Correction

\title{
Correction: Xiong, Z., et al. Different Roles of GRP78 on Cell Proliferation and Apoptosis in Cartilage Development. Int. J. Mol. Sci. 2015, 16, 21153-21176
}

\section{Zhangyuan Xiong ${ }^{1}$, Rong Jiang ${ }^{2}$, Xiangzhu Li ${ }^{1}$, Yanna Liu ${ }^{1}$ and Fengjin Guo ${ }^{1 \text {,* }}$}

Received: 9 November 2015; Accepted: 23 November 2015; Published: 17 December 2015

1 Department of Cell Biology and Genetics, Core Facility of Development Biology, Chongqing Medical University, Chongqing 400016, China; zyxiong38@sina.com (Z.X.); lixzh11@sina.com (X.L.); yanlii@126.com (Y.L.)

2 Laboratory of Stem Cells and Tissue Engineering, Chongqing Medical University, Chongqing 400016, China; Rongjiang86@sohu.com

* Correspondence: guofengjin919@vip.sohu.com or guo.fengjin@gmail.com; Tel.: +86-23-6848-5868; Fax: +86-23-6848-5555

The authors wish to replace Figure 4A on Page 21161 of their paper published in IJMS [1]. There was an error in the representative image of BMP2 + Ad-GRP78 group of C3H10T1/2 in the Figure 4A. The images related to ATDC5 was repeated to show the BMP2 + Ad-GRP78 effects on C3H10T1/2. Please see the corrected Figure 4 here. The authors apologize for any inconvenience. The manuscript will be updated and the original will remain online at the article webpage.

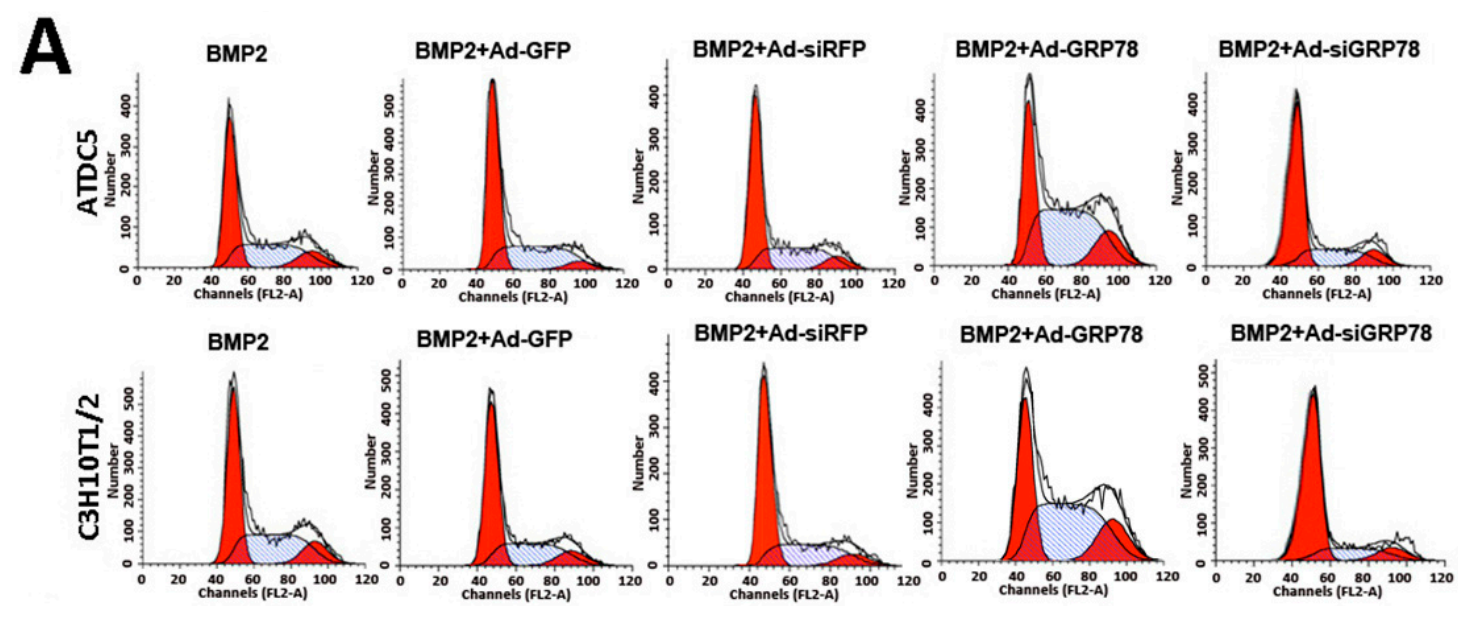

Figure 4. Cont. 
B

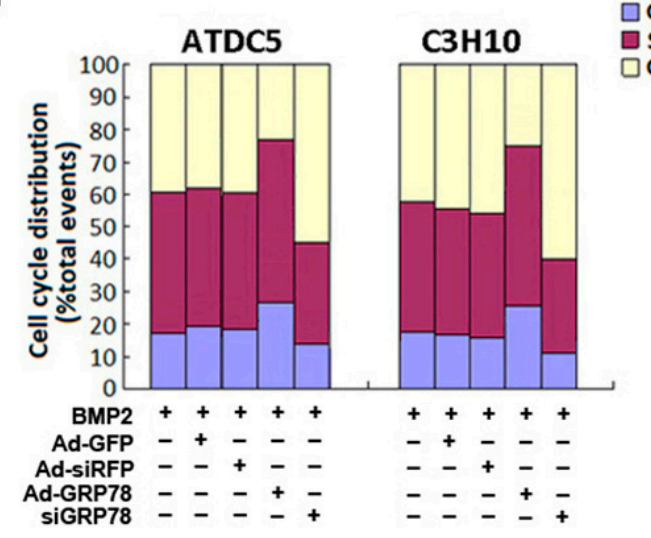

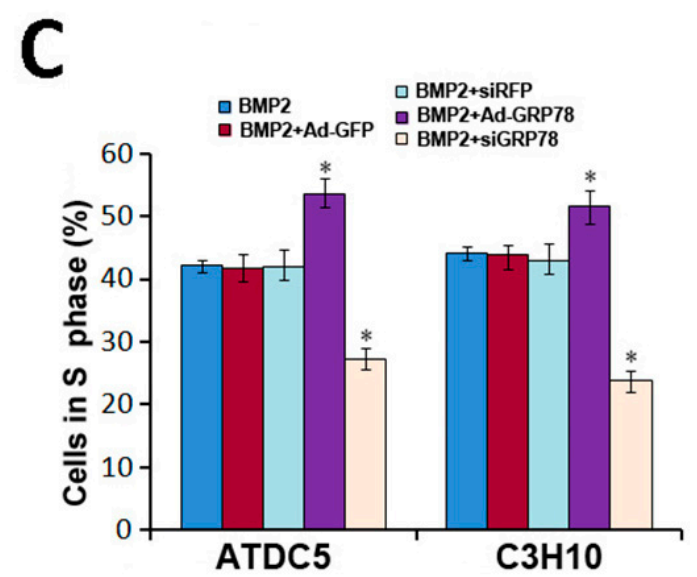

Figure 4. Cellular proliferation analysis by FCM. (A) Flow cytometry images with propidium iodide staining and analysis on cell cycle distribution. Micromass culture of ATDC5 cells and C3H10T1/2 were treated with BMP2 (300 ng/mL) / BMP2 + Ad-GFP/BMP2 + Ad-siRFP / BMP2 + Ad-GRP78 / BMP2 + Ad-siGRP78. Flow cytometry analysis showed that the percentage of the BMP2 + Ad-GRP78 ATDC5 cells in S phase were increased significantly compared to those in BMP2 controls, whereas the percentage of the BMP2 + Ad-siGRP78 ATDC5 cells in S phase were dramatically decreased compared with BMP2 control. The result of $\mathrm{C} 3 \mathrm{H} 10 \mathrm{~T} 1 / 2$ is the same. Experiments were repeated three times, and samples were analyzed by Student's $t$-test and statistical significance with $p<0.05$. Representative images were shown; (B) Flow cytometry assay on the percentages of the ATDC5 and C3H10T1/2 cells in G2/M phase after treatment with BMP2 (300 ng/mL)/BMP2 + Ad-GFP / BMP2 + Ad-siRFP / BMP2 + Ad-GRP78 / BMP2 + Ad-siGRP78; (C) Flow cytometry analysis showed that the percentages of the ATDC5 and C3H10T1/2 Ad-GRP78 cells in S phase were increased significantly, whereas the percentages of the ATDC5 and C3H10T1/2 Ad-siGRP78 cells in S phase were decreased compared with those in their controls. ${ }^{*} p<0.05$ compared with control.

\section{Reference}

1. Xiong, Z.; Jiang, R.; Li, X.; Liu, Y.; Guo, F. Different roles of GRP78 on cell proliferation and apoptosis in cartilage development. Int. J. Mol. Sci. 2015, 16, 21153-21176. [CrossRef] [PubMed]

(C) 2015 by the authors; licensee MDPI, Basel, Switzerland. This article is an open access article distributed under the terms and conditions of the Creative Commons by Attribution (CC-BY) license (http://creativecommons.org/licenses/by/4.0/). 